\title{
Development of an integrated CMOS detector for radon activity measurement and neutron dosimetry
}

\author{
S. Higueret ${ }^{\mathrm{a}}$, M. Trocmé, D. Husson, T.D. Lê, and A. Nourreddine \\ Institut Pluridisciplinaire Hubert Curien (IPHC-DRS), Université Louis Pasteur (ULP), CNRS, IN2P3, 23 rue du Lœss, \\ 67037 Strasbourg, France
}

\begin{abstract}
The development of pixel CMOS sensors for particle tracking in high energy physics has led to promising applications for dosimetry. We present the last results in electronic monitoring of atmospheric radon with the new system-on-chip AlphaRad, as well as highly efficient detection of fast neutrons with a megapixel Active Pixel Sensor of the MIMOSA generation.
\end{abstract}

\section{Introduction}

The IPHC at Strasbourg (Institut Pluridisciplinaire Hubert Curien) has a world-class expertise in CMOS pixel sensors for charged particles tracking [1]. These VLSI monolithic sensors proved high efficiency for charged particles $(>99 \%)$, high spatial resolution $\left(\sigma_{\mathrm{xy}} \sim 1 \mu \mathrm{m}\right)$, high speed ( $\left.5 \mathrm{MHz} / \mathrm{pix}\right)$, low power and low cost (industrial processes with commercial silicon). These pixel devices will be used in the next generation of vertex detectors [1].

Turning to dosimetry applications, we present here the detection of $\alpha$-particles (atmospheric ${ }^{222} \mathrm{Rn}$ ) and fast neutrons through proton recoils with a CMOS pixel chip. Devoted especially to these applications, a new system-on-chip in 0.6 micron CMOS technology has also been designed at the IPHC. The AlphaRad chip [2], with an active area of $5 \times 5 \mathrm{~mm}^{2}$ and working up to $300 \mathrm{kHz}$, shows nearly $100 \%$ efficiency with alpha emitting sources and a remarkably high counting rate of $1.2 \mathrm{MBq} / \mathrm{cm}^{2}$. Both chips are used at room temperature.

\subsection{Application to atmospheric radon}

The first promising application of the AlphaRad chip is the monitoring of atmospheric radon with an inexpensive and fully electronic system. The present prototype is based on independent AlphaRad chips mounted on a printed-circuit board of the same size as non-electronic monitors currently used for radon survey (electrets), typically $6 \mathrm{~cm}$ diameter $\times$ $3 \mathrm{~cm}$ height. The electronic board includes a numeric block based on a Xilinx processor. In a fully passive counting mode, the device detects the total alpha-activity of the neutral ${ }^{222} \mathrm{Rn}$ plus its two daughters ${ }^{218} \mathrm{Po}$ and ${ }^{214} \mathrm{Po}$. The system is already twenty times faster than a previously published experiment [3] based on CMOS pixels, and an excellent linearity has been demonstrated on a special test bench for high radon activities. The future Electronic Radon Monitor will include collection of the Po daughters, providing radon monitoring at low voltage conditions $(5 \mathrm{~V})$, long-term and standalone operation without any external PC.

\footnotetext{
${ }^{a}$ Presenting author, e-mail: higueret@in2p3.fr
}

\subsection{Application to fast neutrons}

Neutron detection is the second application, as CMOS sensors present another feature of extremely high interest: a nearly complete $\gamma$-transparency. From experiments with a calibrated fast neutron source of ${ }^{241} \mathrm{AmBe}$ and with a polyethylene radiator, a very good efficiency of $1.5 \times 10^{-3} \mathrm{p} / \mathrm{n}$ has been obtained. The future detector will have real-time response and $\gamma$-transparency, high efficiency in a small volume at a reasonable cost. The numerous applications in the field of neutron detection include dosimetry in nuclear plants or radioactive waste facilities, at medical cyclotrons as well as neutron beams.

\section{From pixels to the AlphaRad chip}

Since 1999, integrated Active Pixel Systems have shown excellent features for vertexing purposes [1]. The first application to dosimetry was performed with a true CMOS pixel sensor [2], leading to the development of a dedicated chip: the AlphaRad chip is manufactured with a $14 \mu \mathrm{m}$ thick epitaxial layer on a standard silicon substrate, allowing efficient detection of all charged particles able to cross the $\mathrm{SiO}_{2}$ passivation layer $(6 \mu \mathrm{m}$ thick). Charge collection is obtained by pure diffusion of carriers (in less than $500 \mathrm{~ns}$ ) followed by detection on an array of micro-diodes distant of $80 \mu \mathrm{m}$. As the 2048 diodes are connected in parallel, a very fast detection cycle is possible, up to $300 \mathrm{kHz}$ for the full area $\left(2.5 \times 5 \mathrm{~mm}^{2}\right)$ with a negligible dead time. Thanks to a signal/noise ratio of 70 , the measured efficiency [3] of the AlphaRad chip to $5 \mathrm{MeV}$ $\alpha$-particles is close to $100 \%$.

\section{Electronic radon monitoring}

The very first detection of atmospheric radon by a CMOS pixel sensor [2] was published in 2004, establishing the proof-ofprinciple of this measurement. However, the small sensitive area of the MIMOSA-I detector $\left(1.2 \times 1.2 \mathrm{~mm}^{2}\right)$ implies a several-days long recording for typical indoor activities $\left(\sim 1 \mathrm{kBq} / \mathrm{m}^{3}\right)$. Observing that the true pixel structure is not 
really needed for this application, the dedicated system-onchip AlphaRad [3] was designed with a larger sensitive area and a fully parallel readout with a single numeric output, allowing a fast reset cycle up to $300 \mathrm{kHz}$, a promising feature for neutron beam monitoring (see section 4). For radon purposes, we present here a nearly complete device with several independent AlphaRad chips running in parallel on a portable board, ready for a fully standalone mode of operation.

\subsection{Hardware}

In the present configuration, the printed circuit board of $5.2 \mathrm{~cm}$ diameter supports three AlphaRad chips running in parallel, and a complete processing of the analog signal (allowing pileup separation) is performed through a Xilinx FPGA, after analog-to-digital conversion with an 8-bits ADC (32 MHz). To avoid unwanted couplings between the two independent matrices of each chip, a single matrix per chip is used here, thus a total sensitive area of $\mathrm{S}=3 \times\left(5 \times 2.5 \mathrm{~mm}^{2}\right)$.

\section{RS ALP3}

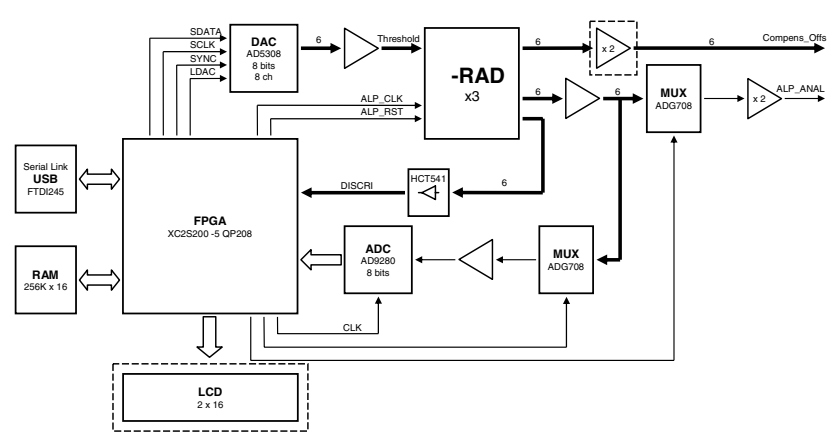

Fig. 1. Synoptic of the readout board for three AlphaRad chips running in parallel.

\subsection{Linearity tests}

Low activity: The electronic board is inserted into the closed tank of $0.224 \mathrm{~m}^{3}$ used at the IPHC, together with an ionisation chamber (IC) as a control device. The ${ }^{222} \mathrm{Rn}$ gas, injected from a standard ${ }^{226} \mathrm{Ra}$ source, has a mean activity of $\mathrm{A}_{\mathrm{v}}=$ $1100 \mathrm{~Bq} / \mathrm{m}^{3}$ (typical indoor critical level). As no filtration of the daughter elements of $\mathrm{Rn}$ is performed, the detected alpha activity is the sum of three emitters, ${ }^{222} \mathrm{Rn}$ plus the two shortlived ${ }^{218} \mathrm{Po}$ and ${ }^{214} \mathrm{Po}$ [2]. For this volumic activity $\mathrm{A}_{\mathrm{v}}$, the measured surface counting rate (in a fully passive counting mode, e.g., without forced air circulation) is $\mathrm{A}_{\mathrm{s}}=6.57 \pm$ $0.40 \alpha /$ h (figure 2 ). This reproducible figure, already twenty times higher than the previously published one [2], will be enhanced again with the next AlphaRad chip (with an active area of $0.5 \mathrm{~cm}^{2}$ per chip).

We define hereafter a Conversion factor to Volumic Activity (labelled $F$ ) through the relationship $A_{v}=F \cdot A_{s}$. This CVA may be expressed in different units, e.g., $\mathrm{kBq} / \mathrm{m}^{3}$ for a given area, and a sampling time which has to be precisely defined. For a better comparison with the ionisation chamber, we choose to express our results in the same acquisition step $\left(\Delta \mathrm{t}_{\mathrm{IC}}=10 \mathrm{~min}\right)$. With this convention, we write our counting rate $A_{\mathrm{s}}=1.095 \pm 0.067\left(\Delta \mathrm{t}_{\mathrm{sampl}}=10 \mathrm{~min}\right)$ for $1.1 \mathrm{kBq} / \mathrm{m}^{3}$, hence a CVA of $F=1.03 \pm 0.06 \mathrm{~m}^{-1}\left(\Delta \mathrm{t}_{\mathrm{sampl}}=10 \mathrm{~min}, \mathrm{~S}=\right.$ $0.375 \mathrm{~cm}^{2}$ ).

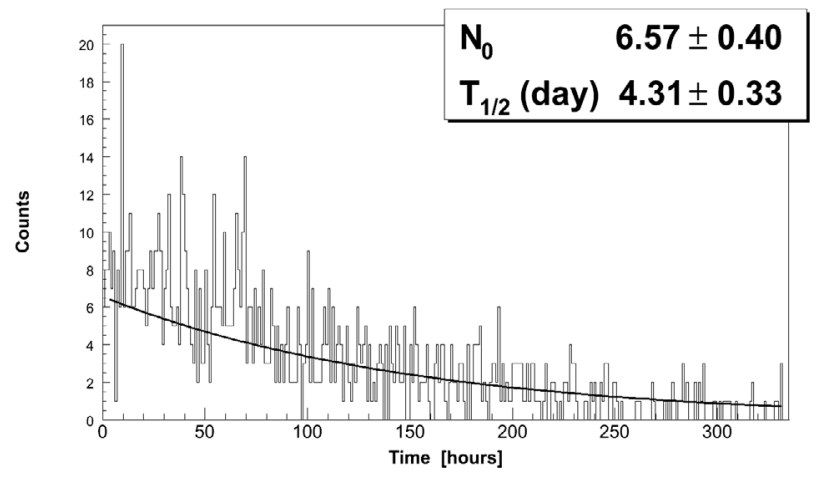

Fig. 2. A ten days fit of the recorded data at $1100 \mathrm{~Bq} / \mathrm{m}^{3}$, showing the large detection fluctuations and the decay of Rn.

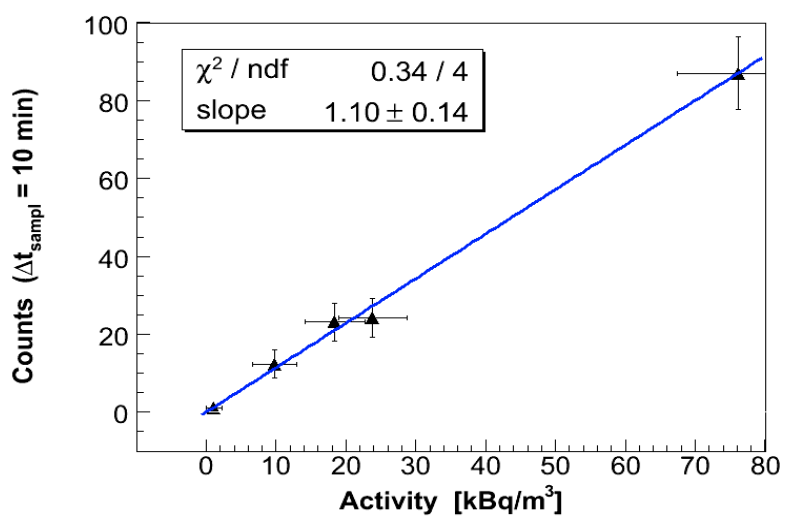

Fig. 3. Linearity of the 3-chips board, in the low activity IPHC tank (first point at $1.1 \mathrm{kBq} / \mathrm{m}^{3}$ ) cumulated with four measurements in the high activity BACCARA bench (from $10-80 \mathrm{kBq} / \mathrm{m}^{3}$ ).

High activity: For a satisfactory test of linearity, better conditions were required, as the IPHC test bench offers a single volumic activity (of only $1100 \mathrm{~Bq} / \mathrm{m}^{3}$ ) and requires several days of delay for regeneration of the $\mathrm{Ra} / \mathrm{Rn}$ source. The LPMA laboratory of the IRSN (Institut de Radioprotection et de Sûreté Nucléaire) in Saclay (France) is equipped with a test bench of large volume $\left(1 \mathrm{~m}^{3}\right)$ and high radon activities [4]. This facility offers the same conditions as the IPHC Strasbourg (slow Rn decay in closed tank) or, on request, constant radon concentration. The main result (figure 3 ) is an excellent linearity (correlation coefficient of 0.95 ) over the range $1.1 \mathrm{kBq} / \mathrm{m}^{3}$ to $80 \mathrm{kBq} / \mathrm{m}^{3}$. The least squares linear fit gives a comparable value for the CVA factor: $F^{\prime}=1.15 \pm$ $0.15 \mathrm{~m}^{-1}\left(\Delta \mathrm{t}_{\mathrm{sampl}}=10 \mathrm{~min}\right)$. 


\section{Fast neutrons}

Our CMOS sensors are able to detect neutrons over the full range of energies, as they may be converted into $\alpha / \mathrm{t}$ particles (through $\mathrm{B} / \mathrm{Li}$ foils) or into recoil protons in $(\mathrm{n}, \mathrm{p})$ elastic reactions on hydrogen-rich converters. Besides fast response and high efficiency to charged particles, these sensors offer the nice feature of being almost free of $\gamma$-contamination. The following measurements were carried out at another IRSN facility: the LDME (Laboratoire de Dosimétrie et Mesure de Neutrons) located in Cadarache (France).

\subsection{Experimental set-up}

The radiator is a polyethylene foil of $0.9 \mathrm{~mm}$ thickness glued in front of an aluminium box containing the MIMOSA V chip [1]. With $512 \times 512$ pixels $17 \mu \mathrm{m}$ apart, the sensitive area is of slightly less than $1 \mathrm{~cm}^{2}$. The acquisition system is based on a local board including a FPGA driven by an external PC. Consecutive frames (of 262144 pixels each) are subtracted two by two, in order to cancel the fixed pattern noise, and an adjustable threshold allows to reduce the recorded data flow which otherwise would be of $10.5 \mathrm{MBytes} / \mathrm{s}$. From preliminary off-source runs, one measures the noise distribution of each pixel. The threshold is set well above five standard deviations of the mean noise. The "Van Gogh" irradiator at Cadarache provides a calibrated neutron field from a $10 \mathrm{Ci}$ AmBe source, installed on a measurement plateau located at $3.2 \mathrm{~m}$ above ground to avoid reflected neutrons. Data from the ISO-certified AmBe source were taken at several distances between $40 \mathrm{~cm}$ and $100 \mathrm{~cm}$ (with laser-monitoring of the source to detector distance).

\subsection{Results}

Fluence: The cluster-search algorithm starts with the recorded pixel showing the highest detected charge, then all neighbouring pixels with a charge at least 5 times greater than their RMS noise are included, and the total charge is compared

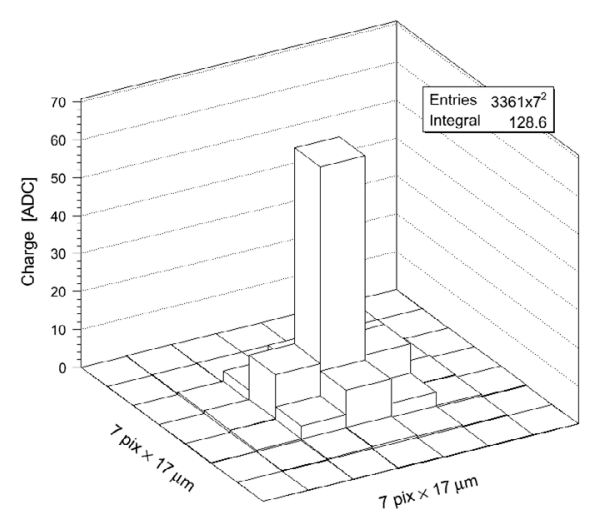

Fig. 4. The typical charge cluster from the recoil protons converted inside the polyethylene and detected by the CMOS pixels. 3361 clusters are averaged here. to the mean noise inside the cluster to compute the signal to noise ratio (SNR). Figure 4 shows the typical cluster of adjacent pixels corresponding to the charge deposited by a single recoil proton hitting the epitaxial layer of the CMOS sensor. On average protons impinge the sensor at perpendicular incidence. At $75 \mathrm{~cm}$ distance, the sensor is crossed by a flux of $528 \mathrm{n} /\left(\mathrm{s} . \mathrm{cm}^{2}\right)$, but only the fastest neutrons of the AmBe spectrum $\left(1.9 \mathrm{MeV}<\mathrm{E}_{\mathrm{n}}<11 \mathrm{MeV}\right)$ generate visible proton, that is, protons able to escape the converter, cross the air layer separating the converter from the sensor and cross and $\mathrm{SiO}_{2}$ layers.

Straggling: At these energies, the elastic (n,p) diffusion is isotropic in the center-of-mass, and simulation indicates that the mean diffusion angle of the protons is $27^{\circ}$ (from the neutron trajectory). Indeed, we could observe some isolated proton tracks at large angles, crossing in this case several adjacent pixels [5]. The cumulated detected charge follows a Landau distribution (straggling), as expected from $\mathrm{MeV}$ protons loosing energy a thin silicon layer (fig. 5).

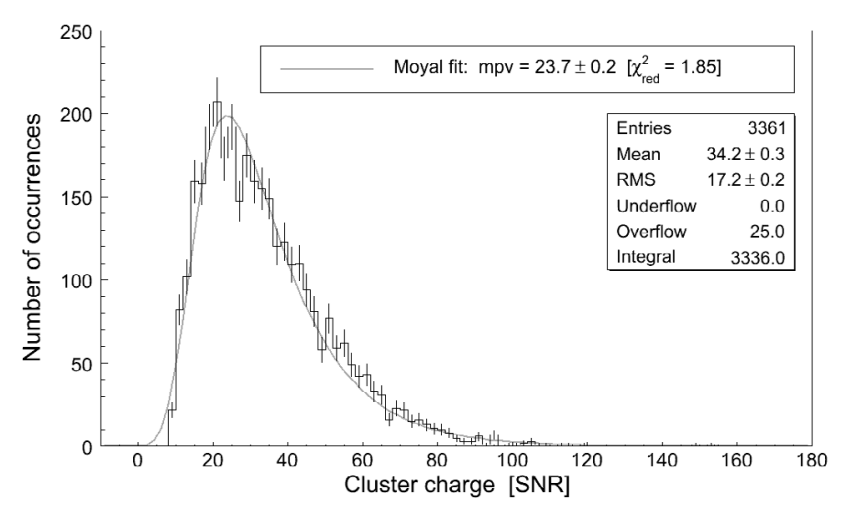

Fig. 5. The observed charge statistics, following the theoretical Landau distribution for thin detectors, and expressed here as a signal to noise ratio.

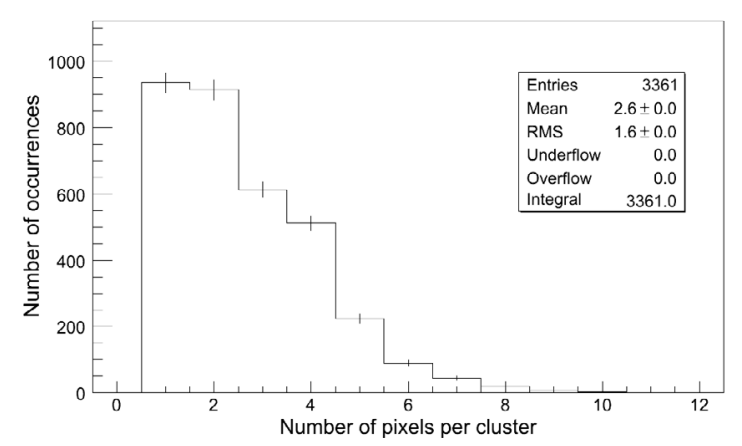

Fig. 6. The pixels-multiplicity distribution inside clusters, here at all cumulated distances.

Multiplicity: The distribution of the pixel multiplicity in each cluster is another figure of interest. The detected protons have a mean multiplicity of 2.5 pixels for a $5 \sigma$ SNR cut, this multiplicity being quite uniform upon the whole detector. The observed data does not exactly follow the theoretical Poisson distribution (fig. 6), suggesting the presence of parasitic 


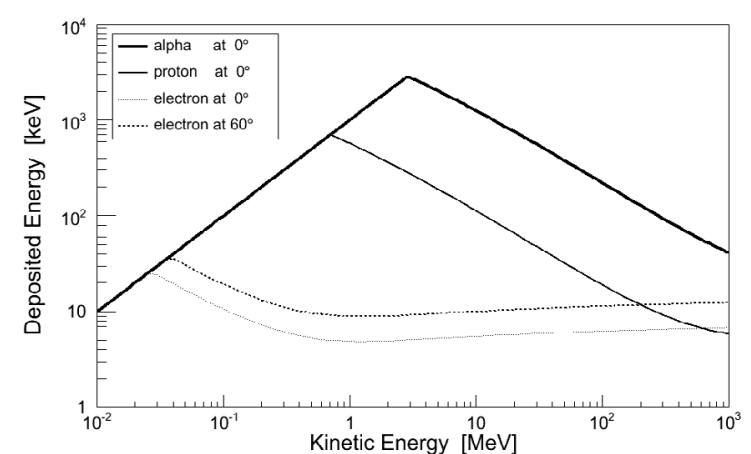

Fig. 7. A Bethe-Bloch calculation showing the excellent rejection of photoelectrons, even for the worst incident angle $\left(60^{\circ}\right.$, corresponding to the $14 \times 17 \times 17 \mu \mathrm{m}^{3}$ pixel diagonal): in the energy range of interest (a few tens of $\mathrm{keV}$ set by the acquisition threshold to $10 \mathrm{MeV}$ ), the signal from protons or $\alpha$-particles is always ten times above that of secondary electrons.

events, as can also be deduced from the efficiency analysis (see next section).

Photon contamination: It could be tempting to consider $\gamma$-photons as an important source of excess events, as we have roughly one photon per neutron in this kind of source. The photon dose $\mathrm{H}^{*}(10)$ at $75 \mathrm{~cm}$ was measured to be 21 $\pm 2 \mu \mathrm{Sv} / \mathrm{h}$. However, in a CMOS detector of only $14 \mu \mathrm{m}$ sensitive thickness, we can safely rule out the presence of photon-induced events, even if one takes into account lowionising electrons generated in the close vicinity of the sensor (aluminium box) by $\mathrm{MeV}$ photons. This very nice feature can be seen with a Bethe-Bloch calculation (fig. 7).

\subsection{Efficiency}

The most important parameter of such a neutron detector is its intrinsic efficiency, calculated as the ratio of detected protons over the flux of neutrons crossing it. Diffusion cross-section values and proton range calculations (in $\left(\mathrm{CH}_{2}\right)_{\mathrm{n}}$, air, $\mathrm{SiO}_{2}$ and $\mathrm{Si}$ ) show that only the last $85 \mu \mathrm{m}$ of the polyethylene radiator can contribute to detectable protons, which must have a minimal energy of $950 \mathrm{keV}$ to reach the sensitive volume [5]. From these considerations, the expected efficiency of our system (in the AmBe spectrum) should be of about $1.1 \times$ $10^{-3} \mathrm{p} / \mathrm{n}$. A more detailed Monte Carlo calculation with the MCNPX code leads again to $\varepsilon_{\mathrm{MC}}=1.1 \times 10^{-3} \mathrm{n} / \mathrm{p}$ [5]. Both calculations do not take into account inelastic reactions inside the detector itself $\left(\mathrm{SiO}_{2}\right.$ and $\left.\mathrm{Si}\right)$ and the surrounding $\mathrm{Al}$ box.

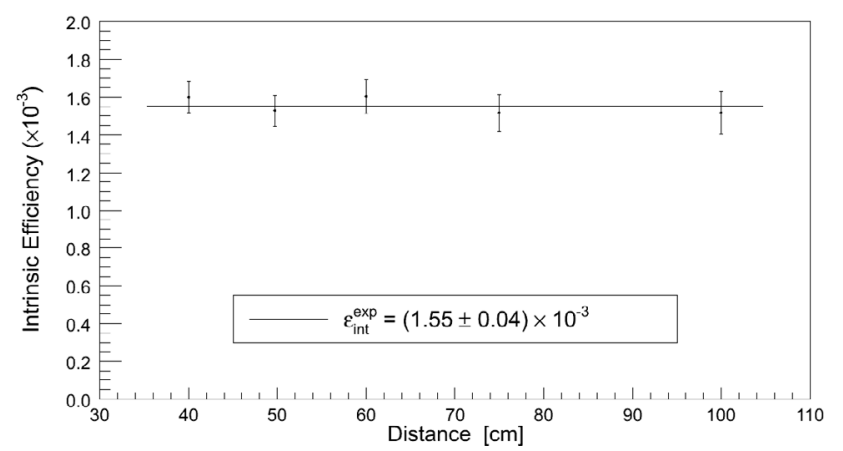

Fig. 8. The measured detection efficiency of the polyethylene/CMOS pixel system for fast neutrons from an AmBe source, over five distances.

Cumulating now all our data at all five distances, we obtain the slightly higher value of $\varepsilon_{\text {data }}=(1.55 \pm 0.04) \times 10^{-3}$. The mentioned error is purely statistic, and obviously, we have systematic contributions from the sensor itself (some lack of homogeneity), from inelastic scattering $((n, p)$ reactions in $\mathrm{Si}$ and the $\mathrm{Al}$ box) as well as scattered neutrons inside the box. More detailed simulations are underway, but at this stage, the measured efficiency is in good agreement with the calculated values, making this first achievement really promising.

We gratefully acknowledge N. Michielsen and S. Bondiguel (IRSN/DSU/SERAC/LPMA) for their kind help at the BACCARA facility in Saclay. We are also greatly indebted to L. Van Ryckeghem and G. Pelcot for efficient support at Cadarache, as well as to C. Dominingo for his precious help with MCNPX.

\section{References}

1. R. Turchetta, D. Husson et al., Nucl. Instrum. Meth. A 473, 83 (2001).

2. A. Nachab, D. Husson, S. Higueret et al., Nucl. Instrum. Meth. B 225, 418 (2004).

3. D. Husson, A. Bozier, S. Higueret, T.D. Lê, A. Nourreddine, Nucl. Instrum. Meth. A 569, 845 (2006).

4. BACCARA testing facility at the IRSN institute of Saclay (DSU/SERAC/LPMA) http://www.irsn.org/radon.

5. M. Trocmé, S. Higueret, D. Husson, T.D. Lê, A. Nourreddine, in Proceedings of the tenth symposium on neutron dosimetry, Uppsala, 2006 (to be published in Radiation Protection Dosimetry). 